\title{
Geração de zonas de manejo para cafeicultura empregando-se sensor SPAD e análise foliar ${ }^{1}$
}

\author{
Francelino A. Rodrigues Junior ${ }^{2}$, Luciano B. Vieira ${ }^{3}$, D aniel M. de Q ueiroz ${ }^{3} \&$ Nerilson T. Santos ${ }^{4}$
}

\begin{abstract}
RESUMO
O bjetiva-se, com este trabalho, definir zonas de manejo para cafeicultura por meio dos métodos $\mathrm{K}$ M eans e Fuzzy C-M eans, com base em determinações realizadas com sensor de clorofila e por análise foliar, e avaliar as zonas de manejo obtidas usando-se os dois métodos de agrupamento. 0 trabalho foi desenvolvido na Fazenda Jatobá, localizada no município de Paula Cândido, MG, durante o mês de novembro de 2007, enquanto a lavoura se apresentava no estado "chumbinho". A área avaliada apresenta uma lavoura de Coffea arabica L. Cv. Catuaí de 2,1 ha. Foram definidas, por meio dos dois métodos propostos, as zonas de manejo, com base nas seguintes análises: Valores de SPAD; concentrações foliares de N, P e K; concentrações foliares de $\mathrm{N}$ e $\mathrm{Ca}$; concentrações foliares de $\mathrm{N}$; concentrações foliares de N, Zn e B; concentrações foliares de N, P, K, Ca e S e concentrações foliares de N, Ca e S. O s métodos de agrupamento de dados K-M eans e Fuzzy C-M eans não apresentaram diferenças na geração das zonas de manejo. Houve baixa similaridade entre as zonas de manejo geradas com uso do SPAD e concentrações foliares.
\end{abstract}

Palavras-chave: análise de agrupamento, clorofilômetro, nutrientes foliares

\section{Coffee crop management zone delimitation using chlorophyll index and leaf analysis}

\begin{abstract}
The objective of this work was to define management zones for fertilizer application in coffee crop by using the K-means and the Fuzzy C-Means methods. The data used to define management zones were the chlorophyll index measured by SPAD sensor and the nutritional coffee leaf analysis performed in laboratory. The study was conducted in Jatoba Farm, located in Paula Candido, M inas Gerais state, Brazil. The data was collected in N ovember, 2007 when the coffee fruits were starting their development. The coffee variety was Coffea arabica Catuai, and the total analyzed area was 2.1 ha. The management zones were generated using different set of data: the SPAD values; the N, P and $\mathrm{K}$ leaf concentrations; the $\mathrm{N}$ and $\mathrm{C}$ a leaf concentrations; the $\mathrm{N}, \mathrm{Zn}$ and $\mathrm{B}$ leaf concentrations; the N, P, K, Ca and S leaf concentrations; and the $\mathrm{N}, \mathrm{C}$ a and $\mathrm{S}$ leaf concentrations. The management zones generated by using K-means and Fuzzy $C-M$ eans did not present difference in management zone delimitation. The management zones defined by using the SPAD values were different from the ones generated by using leaf analysis.
\end{abstract}

Key word: clustering analysis, chlorophyll-meter, leaf nutrients

\footnotetext{
1 Parte da Dissertação de Mestrado do primeiro autor defendida na U FV

2 FEAGRI/U N ICAMP, Av. Candido Rondon, 501, CEP 13083-875, Campinas, SP. E-mail: farjunior@hotmail.com

3 UFV/DEA - Departamento de Engenharia Agrícola, Av. P.H. Rolfs, s/ №, Campus U niversitário, CEP 36570-000, Viçosa, M G. Fone: (31) $3899-2729$. E-mail: Ibaiao@ufv.br; queiroz@ufv.br

${ }^{4}$ UFV/DET - Departamento de Estatística, Av. P.H. Rolfs, s/ noo, Campus U niversitário, CEP 36570-000, Viçosa, MG. Fone: (31) $3899-2398$. E-mail: nsantos@ufv.br
} 


\section{INTRODUÇÃO}

A agricultura de precisão pode trazer inúmeros benefícios ao cultivo do café, pois trata-se de uma cultura de elevada receita por área cujo preço se baseia na qualidade dos grãos. O emprego das técnicas de agricultura de precisão pode auxiliar na identificação de áreas com potencial para produção de frutos com melhor qualidade e, até mesmo, no entendimento dos fatores que a determinam. As áreas de produção podem ser georreferenciadas e tratadas de maneira diferenciada uma vez que a cultura é perene e vem sendo cultivada tradicionalmente em talhões com áreas relativamente pequenas (Queiroz et al., 2004).

Um dos problemas-chave da agricultura de precisão é a necessidade de uma grade de amostragem densa, a fim de se detectar a variabilidade espacial do atributo em estudo e possibilitar a geração de mapas que representem, de maneira real, tal área. Uma das soluções para este impasse vem sendo a geração de zonas de manejo.

Uma zona de manejo é definida como uma sub-região do campo que apresenta uma combinação de fatores limitantes de produtividade e de qualidade para a qual se pode aplicar uma dose uniforme de insumos. A definição de zonas de manejo torna mais fácil a aplicação das técnicas de AP uma vez que se pode empregar, no manejo das culturas, os mesmos sistemas utilizados na agricultura convencional.

Sabe-se que a experiência dos produtores foi extremamente importante no desenvolvimento da agricultura, visto que eles sabem identificar quais áreas de uma cultura apresentam maiores e menores rendimentos. O uso desta base de conhecimento pode permitir a identificação de diferentes zonas de manejo com base na história da produção (Fleming \& Westfall, 2000). Técnicas para análises de agrupamento de dados têm sido usadas para a delimitação de zonas de manejo possibilitando não só o uso de um conjunto de fatores proeminentes do desenvolvimento das culturas mas também identificar variabilidades nos cultivos.

Técnicas de análises de agrupamento de dados têm sido classificadas dentro de dois métodos principais: Hierárquico e Particionado. Os métodos particionados são de grande valia e possibilitam o uso de um conjunto de atributos diretamente correlacionados com a variabilidade nutricional da área em estudo podendo-se, então, tal variabilidade; dentre os métodos se encontram o K-Means e o Fuzzy C-Means (Taylor et al., 2003; 2007; Santos et al., 2003; Yan et al., 2007). Tais zonas de manejo formam um banco de dados o qual pode ser utilizado no intuito de direcionar uma futura amostragem, aumentando as amostras em áreas de maior variabilidade e as diminuindo em áreas mais homogêneas.

Taylor et al. (2003) utilizaram o algoritmo K-means para definir zonas de manejo por meio de dados de produtividade e indução eletromagnética do solo e concluíram que essas zonas coincidem com diferenças em níveis de nutrientes do solo; assim, foi possível formar um banco de dados para a realização de uma amostragem mais direcionada, caso em que o número de amostras de solo foi reduzido de 27 para 4 . O algoritmo Kmeans permite que as variações do solo e produtividade sejam usadas juntas na definição de zonas de manejo possibilitando, ao usuário, controlar o número de zonas identificadas, proporcionando melhor manejo das áreas.

Ortega \& Santibáñez (2007) avaliaram três métodos para delimitação de zonas de manejo com base em dados de fertilidade de solo para comparação com a variabilidade da produtividade em área cultivada com milho. Os métodos comparados foram o de análise de agrupamento (K-means), análise de componentes principais e o método de coeficiente de variação. Os resultados indicaram que os três métodos foram similares e adequados para delimitar zonas de manejo homogêneas em relação à fertilidade do solo; no entanto, na maioria das áreas, $77 \%$, os métodos utilizados para delimitação das zonas de manejo foram incapazes de detectar níveis de produtividade.

Modelos "fuzzy" têm sido utilizados em projetos de sistemas de controle. Algoritmos de agrupamentos "fuzzy" são ferramentas matemáticas utilizadas para detectar similaridade entre membros de uma coleção de objetos (Windham, 1982; Ateca et al., 2001).

Yan et al. (2007), utilizando ferramentas da geoestatística e o algoritmo de agrupamento de dados fuzzy c-means na geração de zonas de manejo para a caracterização das propriedades químicas do solo em terras salinas, concluíram que as zonas geradas se mostraram favoráveis ao conhecimento da variabilidade na produtividade. Tais resultados provêm uma base de informações para o manejo mais racional e preciso, podendo ser utilizados para o desenvolvimento de uma amostragem mais direcionada e menos uniforme, focando os locais de maior variabilidade, o que possibilita a identificação das propriedades químicas do solo que influenciam na produtividade, minimizando o custo em análises de solo, que é o problema-chave da agricultura de precisão.

Visto que o problema-chave da agricultura de precisão é a necessidade de uma grade amostral densa, a correlação das zonas de manejo obtidas por meio dos valores SPAD e concentrações de nutrientes foliares, podem levar a um entendimento melhor da variabilidade nutricional. Referidas zonas de manejo podem ser utilizadas como orientação para futuras amostragens, facilitando a otimização desta operação.

Desta forma, o objetivo geral deste trabalho foi a geração de zonas de manejo através dos métodos K-means e Fuzzy Cmeans para a cafeicultura, com base em determinações realizadas com sensor de clorofila e na análise foliar.

\section{Material e MÉTOdos}

Este trabalho foi conduzido na Fazenda Jatobá, localizada no município de Paula Cândido, MG, nas coordenadas geográficas de $20^{\circ} 49^{\prime} 45,9^{\prime \prime} \mathrm{S}$ e $42^{\circ} 55^{\prime}$ '5,7' O, durante o mês de novembro de 2007. Os frutos da cultura se apresentavam no estado "chumbinho". A área avaliada se refere a uma lavoura de Coffea arabica L. cv. Catuaí de 2,10 ha irrigada por um pivôcentral, em solo caracterizado como Argissolo VermelhoAmarelo. O espaçamento da cultura é de 0,65 m entre plantas e 3,0 m entre linhas, totalizando 26 linhas de plantio. 
Iniciou-se o trabalho com o georreferenciamento da área em estudo, utilizando-se o aparelho DGPS modelo ProXRS, fabricado pela Trimble. Para fazer a correção diferencial do levantamento utilizaram-se os dados da base da RBMC (Rede Brasileira de Monitoramento Contínuo) do IBGE, localizada na UFV e o programa computacional Pathfinder Office da Trimble, para correção dos dados.

A amostragem para a coleta dos valores de SPAD foi feita de forma alternada em relação às linhas de plantio nas quais foi amostrada uma planta, a cada quatro plantas, totalizando $25 \%$ de todas as plantas presentes nas linhas e 1141 plantas, aproximadamente $13 \%$ das plantas presentes na área. A coleta de folhas submetidas à análise foliar foi realizada a cada 10 plantas usadas para leitura SPAD; logicamente, esta décima planta constou de determinações simultâneas de SPAD e valores da análise foliar, totalizando 114 plantas.

Cada planta amostrada foi dividida ao meio em relação ao sentido da linha; foram medidos os valores de SPAD em 15 pares de folhas em ramos produtivos (terceiro ou quarto par de folhas, a partir do ápice do ramo) no terço médio da planta, totalizando 30 folhas por planta, 15 folhas de cada lado; anotouse o valor médio dos dois lados, todos medidos no terço médio da planta. As análises foliares foram feitas no Laboratório de Análise de Solo Viçosa Ltda utilizando-se o método de "Kjeldahl" para determinação do N, incineração em estufa a $500{ }^{\circ} \mathrm{C}$ para determinação do boro e, para os demais nutrientes, a digestão úmida em solução nitro-perclórica.

Dois métodos foram utilizados para definição das zonas de manejo, K-means (KM) e Fuzzy C-means (FCM). Visando à definição dos dados a serem utilizados como base na definição das zonas de manejo, análises geoestatísticas foram feitas no programa computacional GS+ com cada atributo, verificandose sua possível dependência espacial a fim de determinar as concentrações foliares que constituiriam cada conjunto de dados para a geração das zonas de manejo; posteriormente, os atributos de cada conjunto foram dispostos em uma grade padrão de 0,7 x $0,7 \mathrm{~m}$ por meio da krigagem, adaptado de Taylor et al. (2007).

Aplicou-se o método KM para dividir os valores de cada conjunto de dados em três grupos, ou seja, cada mapa temático gerado a partir dos conjuntos de dados possui três zonas de manejo; para isto, o algoritmo é executado iterativamente, da seguinte forma, por meio do programa computacional Statistica 7:

1. Definiram-se os três pontos no espaço, representados pelos objetos que foram agrupados; esses pontos representam os centroides iniciais de cada grupo (zona de manejo).

2. Atribuiu-se a cada objeto o grupo que tem o centroide o mais próximo dele.

3. Quando todos os objetos foram recebidos pela atribuição do grupo a que pertencem, recalcularam-se as posições dos três centroides.

4. Repetiram-se os passos 2 e 3 até que os centroides não mais se movessem.

A função objetivo para o método KM é:

$$
\operatorname{Minimizar} J=\sum_{j=1 i=1}^{k} \sum_{i}^{n}\left\|x_{i}(j)-c_{j}\right\|^{2}
$$

em que: $\left\|\mathrm{x}_{\mathrm{i}}^{(\mathrm{j})}-\mathrm{c}_{\mathrm{j}}\right\|$ é a distância euclidiana entre um vetor de dados $x_{i}^{(j)}$ (de cada conjunto de dados $\mathrm{j}$ ) e o centro do agrupamento $c_{j}$, sendo um indicador da distância de $n$ pontos dos dados para os respectivos centros dos $\mathrm{k}$ agrupamentos.

Cinco testes foram feitos partindo-se de centroides diferentes e se observam que, para todos os centroides iniciais, obteve-se o mesmo resultado.

O método FCM testa subagrupamentos de dados buscando a minimização da variância intraclasses e a maximização da variância interclasses. Por este método faz-se a minimização da função objetivo $\mathrm{J}_{\mathrm{m}}$, com respeito a uma função característica $\mu_{\mathrm{ij}}\left(\mu_{\mathrm{ij}}{ }^{\mathrm{a}}[0,1]\right)$ e um centro de classe $\mathrm{c}_{\mathrm{i}}$. Usou-se este método para dividir, em três classes, os grupos de dados utilizados na confecção das zonas de manejo, razão por que se utilizou o programa computacional FuzMe 3.0 (Minasny \& Mcbratney, 2002). A função objetivo é:

$$
\operatorname{Minimizar} \mathrm{J}_{\mathrm{m}}=\sum_{\mathrm{i}=1 \mathrm{j}=1}^{\mathrm{k}} \sum_{\mathrm{j}=1}^{\mathrm{n}}\left(\mu_{\mathrm{ij}}\right)^{\mathrm{m}} \mathrm{d}^{2}\left(\mathrm{x}_{\mathrm{j}}, \mathrm{c}_{\mathrm{i}}\right)
$$

sendo:

$$
\mathrm{d}^{2}\left(\mathrm{x}_{\mathrm{j}} ; \mathrm{c}_{\mathrm{i}}\right)=\left\|\mathrm{x}_{\mathrm{j}}-\mathrm{c}_{\mathrm{i}}\right\|
$$

em que m é um índice do grau fuzzy para a classificação. Segundo Yonamine et al. (2002), a variável real $\mathrm{m}>1$ é usada para definir a distância permitida entre os pontos e o centro que está sendo calculado. Quanto maior o valor de m mais elementos do conjunto são considerados pertencentes a um grupo. Este parâmetro é escolhido de acordo com o problema considerado, não existindo base teórica alguma para a escolha ótima do valor de m.

Realizou-se, para o presente trabalho, um teste preliminar, em que se testaram cinco diferentes valores de $\mathrm{m}: 1,1 ; 1,3 ; 2 ; 5$ e 10, cujos resultados mostraram que nesta faixa o valor de $m$ não alterou a definição das zonas de manejo e, assim sendo, adotou-se $m=1,1$ para a geração das zonas de manejo.

Como critério de parada da iteração foi recalculada a função $\mathrm{J}_{\mathrm{m}}$ até que a diferença entre $\mathrm{J}_{\mathrm{m}}$ atual e $\mathrm{J}_{\mathrm{m}}$ anterior fosse igual ou menor que 0,0001 . Dez inicializações aleatórias foram feitas com diferentes centroides iniciais.

Após a delimitação dos grupos pelos métodos KM e FCM, utilizou-se o programa computacional ArcGis para a geração dos mapas temáticos.

A partir dos mapas gerados por meio dos dois métodos de agrupamento de dados (KM e FCM) foi analisado, visualmente, aquele que obteve maior similaridade entre as zonas de manejo provenientes do SPAD e as demais zonas geradas.

Para efetuar uma análise quantitativa fez-se uma grade padrão com 100 pontos em cada mapa de zonas de manejo gerado e se analisou, dentre as três classes, para qual delas cada ponto desta grade pertence; verificou-se, então, qual dos dois métodos obteve maior número de pontos de mesma classe entre os mapas comparados além, também, das médias obtidas de cada atributo dentro de cada classe. 
Tais comparações se referem aos mapas gerados com os mesmos atributos comparando-se os dois métodos de agrupamento e os mapas gerados dentro de cada método a partir das concentrações foliares com o mapa gerado e dos valores de SPAD. Através desses parâmetros avaliou-se se houve diferença entre os dois métodos na geração de tais zonas e quais mapas gerados a partir das concentrações foliares obtiveram maior similaridade entre os mapas de SPAD para os dois métodos.

\section{RESULTADOS E DISCUSSÃO}

Na Tabela 1 são apresentadas as análises estatísticas descritivas dos valores SPAD e dos nutrientes e a faixa considerada satisfatória (FS) dos nutrientes quanto aos seus níveis para a cultura do café cultivado em Viçosa, MG, de acordo com Martinez et al. (2003).

A folha expressa, em sua coloração, os efeitos sintomáticos do "status" de nutrientes. Um dos sintomas da deficiência dos nutrientes é a clorose (amarelecimento das folhas); esta clorose ocorre com frequência nas folhas mais velhas quando se dá a deficiência de $\mathrm{N}$ e $\mathrm{Mg}$ e nas folhas mais jovens por ocasião da deficiência de $\mathrm{Ca}$; este sintoma indica, ainda, redução ou aumento na quantidade de clorofila relacionado ao nível desses nutrientes; a quantidade de nutrientes que influencia a síntese de clorofila dificulta a discriminação do efeito isolado de cada nutriente, pelo SPAD.
Observa-se, enquanto os valores de $\mathrm{N}$ estão dentro do nível satisfatório, que os valores de $\mathrm{Ca}$ e $\mathrm{Mg}$ se encontram em baixos níveis. O Mg é parte integrante da molécula da clorofila e só isto basta para mostrar sua importância; além do mais, exerce papel relevante no metabolismo do fósforo; por outro lado, esses nutrientes tendem a diluir o efeito isolado de cada nutriente sobre a quantidade de clorofila presente na folha.

Segundo Lange et al. (2005), tratamentos com e sem omissão de micronutrientes ( $\mathrm{B}, \mathrm{Cu}, \mathrm{Fe}, \mathrm{Mn}, \mathrm{Mo}, \mathrm{Zn}$ ) influenciaram, de modo diferente, o teor de clorofila medido em unidades SPAD ao longo do período experimental, na cultura da mamona, demonstrando a influência que os micronutrientes podem ter no teor de clorofila identificado através do sensor, o que implica na existência de um número maior de fatores que deve ser levado em consideração ao se avaliar o teor de $\mathrm{N}$ nas folhas, através do SPAD.

Todos os atributos foram submetidos à análise geoestatística. Os parâmetros dos semivariogramas ajustados e o coeficiente de determinação da validação cruzada para cada atributo são apresentados na Tabela 2 .

Tendo em vista os valores dos coeficientes de determinação dos semivariogramas $\left(\mathrm{R}_{1}^{2}\right)$, os índices da dependência espacial (IDE) e os coeficientes de determinação da validação cruzada $\left(\mathrm{R}_{2}^{2}\right)$, pode-se observar, na Tabela 2, que os elementos $\mathrm{Mg}, \mathrm{Fe}$, $\mathrm{Cu}$ apresentaram IDE considerados elevados, segundo Zimback (2001), com moderados $\mathrm{R}_{1}^{2}$, porém com baixos valores de $\mathrm{R}_{2}^{2}$. O elemento $\mathrm{Mn}$ indicou valor de IDE moderado e $\mathrm{R}_{1}{ }^{2}$ elevado mas, como os citados acima, baixo valor de $\mathrm{R}_{2}^{2}$.

Tabela 1. Análise descritiva das concentrações de nutrientes foliares e faixa considerada satisfatória

\begin{tabular}{|c|c|c|c|c|c|c|c|c|c|}
\hline Elemento & $\begin{array}{l}\text { Valor } \\
\text { mínimo }\end{array}$ & $\begin{array}{l}\text { Quartil } \\
\text { inferior }\end{array}$ & Mediana & $\begin{array}{c}\text { Quartil } \\
\text { superior }\end{array}$ & $\begin{array}{l}\text { Valor } \\
\text { máximo }\end{array}$ & Média & $\begin{array}{l}\text { Desvio } \\
\text { padrão }\end{array}$ & $\begin{array}{l}\text { Coeficiente de } \\
\text { variação (\%) }\end{array}$ & FS * \\
\hline SPAD & 47,90 & 60,10 & 62,40 & 64,50 & 71,20 & 62,09 & 3,47 & 5,58 & \\
\hline $\mathrm{N}-$ dag $\mathrm{kg}^{-1}$ & 2,69 & 2,88 & 3,02 & 3,11 & 3,42 & 3,01 & 0,17 & 5,64 & $2,47-3,15$ \\
\hline$P$ - dag $\mathrm{kg}^{-1}$ & 0,12 & 0,15 & 0,16 & 0,17 & 0,20 & 0,15 & 0,01 & 6,66 & $0,15-0,19$ \\
\hline $\mathrm{K}$ - dag $\mathrm{kg}^{-1}$ & 1,44 & 1,67 & 1,90 & 2,17 & 2,47 & 1,92 & 0,28 & 14,58 & $2,13-2,89$ \\
\hline $\mathrm{Ca}-$ dag kg ${ }^{-1}$ & 0,48 & 0,76 & 0,88 & 1,02 & 1,28 & 0,89 & 0,18 & 20,22 & $0,92-1,20$ \\
\hline $\mathrm{Mg}-$ dag $\mathrm{kg}^{-1}$ & 0,24 & 0,32 & 0,35 & 0,38 & 0,45 & 0,34 & 0,04 & 11,76 & $0,35-0,56$ \\
\hline $\mathrm{S}-$ dag $\mathrm{kg}^{-1}$ & 0,11 & 0,13 & 0,15 & 0,17 & 0,24 & 0,15 & 0,03 & 20,00 & $0,16-0,22$ \\
\hline $\mathrm{Zn}-\mathrm{mg} \mathrm{kg}^{-1}$ & 6,00 & 8,00 & 8,00 & 9,00 & 12,00 & 8,87 & 1,28 & 14,43 & $6-12$ \\
\hline $\mathrm{Fe}-\mathrm{mg} \mathrm{kg}^{-1}$ & 72,00 & 110,00 & 123,00 & 142,25 & 467,00 & 137,87 & 56,62 & 41,06 & $56-94$ \\
\hline $\mathrm{Mn}-\mathrm{mg} \mathrm{kg}^{-1}$ & 226,00 & 496,00 & 607,50 & 722,75 & 1249,00 & 617,75 & 181,40 & 29,36 & $115-286$ \\
\hline $\mathrm{Cu}-\mathrm{mg} \mathrm{kg}^{-1}$ & 9,00 & 13,00 & 13,00 & 15,00 & 22,00 & 13,80 & 2,26 & 16,37 & $13-29$ \\
\hline $\mathrm{B}-\mathrm{mg} \mathrm{kg}^{-1}$ & 15,70 & 25,80 & 29,40 & 32,70 & 40,00 & 29,20 & 5,36 & 18,35 & $29-52$ \\
\hline
\end{tabular}

* Faixa considerada satisfatória quanto aos níveis de concentração de nutrientes para a cultura do café cultivado em Viçosa, MG

Tabela 2. Parâmetros dos semivariogramas ajustados e coeficiente de determinação da val idação cruzada de cada atributo

\begin{tabular}{lccccccc}
\hline & Modelo & Co & Co+ C & Alcance & $\mathbf{R}_{\mathbf{1}}{ }^{2}$ & [C/(Co+ C)] & $\mathbf{R}_{\mathbf{2}}{ }^{2}$ \\
SPAD & Exponencial & 3,70 & 10,714 & 18,1 & 0,983 & 0,654 & 0,420 \\
$\mathrm{~N}$ & Gaussiano & $1,54110^{-2}$ & $3,52210^{-2}$ & 40,6 & 0,659 & 0,562 & 0,492 \\
$\mathrm{P}$ & Exponencial & $210^{-5}$ & $1,710^{-4}$ & 25,0 & 0,887 & 0,880 & 0,422 \\
$\mathrm{~K}$ & Gaussiano & $3,3310^{-2}$ & $1,11610^{-1}$ & 53,1 & 0,969 & 0,700 & 0,561 \\
$\mathrm{Ca}$ & Gaussiano & $8,5610^{-3}$ & $2,95210^{-2}$ & 13,4 & 0,802 & 0,710 & 0,548 \\
$\mathrm{Mg}$ & Exponencial & $1,510^{-4}$ & $1,7310^{-3}$ & 4,30 & 0,506 & 0,910 & 0,003 \\
$\mathrm{~S}$ & Esférico & $4,610^{-4}$ & $9,510^{-4}$ & 70,2 & 0,926 & 0,510 & 0,235 \\
$\mathrm{Zn}$ & Gaussiano & $1,5310^{-1}$ & 1,69 & 13,6 & 0,867 & 0,900 & 0,522 \\
$\mathrm{Fe}$ & Gaussiano & 1,0 & 2532,0 & 10,0 & 0,354 & 0,990 & 0,028 \\
$\mathrm{Mn}$ & Exponencial & 18060,0 & 36130,0 & 18,5 & 0,820 & 0,500 & 0,069 \\
$\mathrm{Cu}$ & Esférico & $110^{-2}$ & 4,798 & 7,00 & 0,000 & 0,990 & 0,037 \\
$\mathrm{~B}$ & Gaussiano & 9,84 & 33,5 & 32,3 & 0,914 & 0,706 & 0,537 \\
\hline
\end{tabular}

Co: efeito pepita; Co+C: patamar; $\mathrm{R}_{1}{ }^{2}$ : coeficiente de determinação do semivariograma; $[\mathrm{C} /(\mathrm{Co}+\mathrm{C})]$ : índice de dependência espacial; $\mathrm{R}_{2}{ }^{2}$ : coeficiente de determinação da validação cruzada 
Com a validação cruzada é possível identificar áreas em que não houve dependência espacial; para essas áreas, novas amostragens podem ser necessárias (Isaaks \& Srivastava, 1989) mostrando, desta forma, a importância da validação cruzada para indicar a robustez do modelo escolhido na estimação de valores em locais dos quais não se possui informação.

Assim e com base nos resultados de $\mathrm{R}_{2}{ }^{2}$ obtidos, os elementos $\mathrm{Mg}, \mathrm{Mn}$, Fe e Cu não foram utilizados para definição das zonas de manejo enquanto os demais elementos forneceram valores acima de 0,5 para IDE e $R_{1}^{2}$, e acima de 0,2 para $R_{2}^{2}$, possibilitando serem utilizados na geração das zonas de manejo; desta forma se definiram as zonas de manejo, com base nos seguintes dados: valores de SPAD; concentrações foliares de N; concentrações foliares de N, P e K; concentrações foliares de $\mathrm{N}, \mathrm{Ca}$; concentrações foliares de N, Zn e B; concentrações foliares de N, P, K, Ca e S; concentrações foliares de N, Ca e S.

Na Figura 1 se acham as zonas de manejo geradas a partir dos valores de SPAD, pelos métodos KM e FCM.

A.

B.
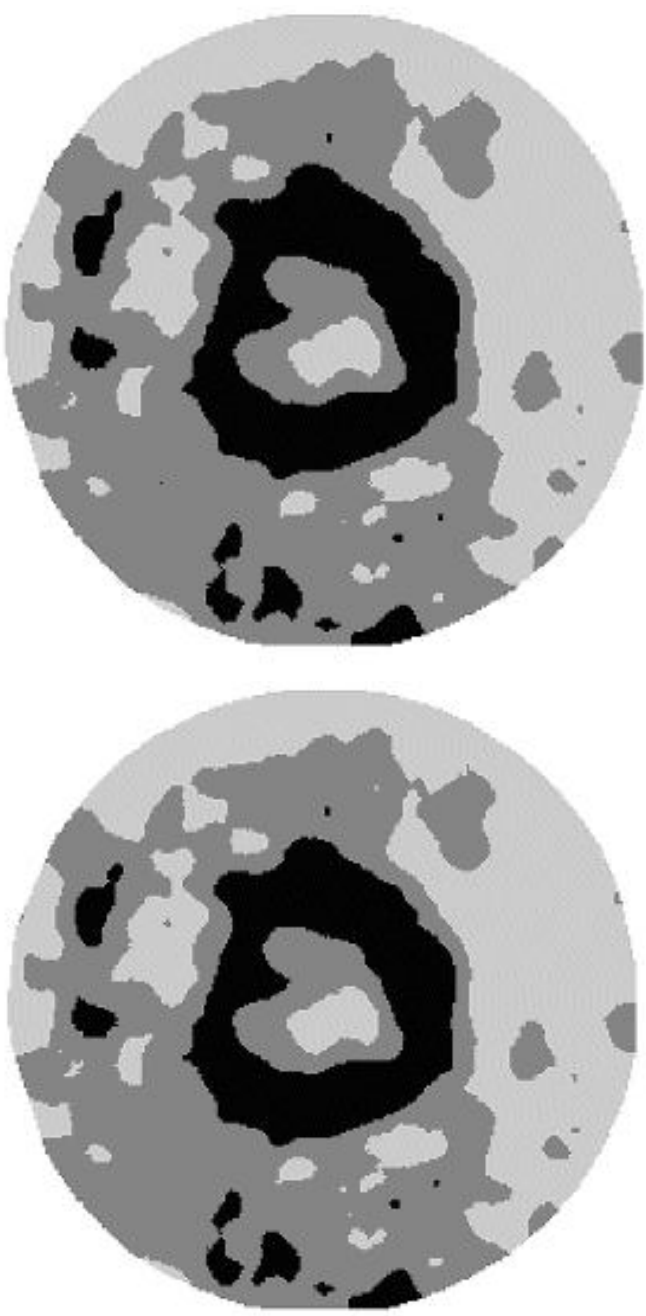

Classes

Figura 1. Zonas de manejo geradas a partir dos valores de SPAD, pelos métodos KM (A) e FCM (B)
Na Tabela 3 se tem os valores médios de SPAD que cada classe representa nas zonas de manejo geradas para os métodos KM e FCM e quantos pontos da grade padrão de 100 pontos pertencem a cada grupo.

Tabela 3. Valores médios de SPAD e número de pontos da grade padrão de cada classe das zonas de manejo obtidas pelos métodosKM e FCM

\begin{tabular}{cccccc}
\hline \multirow{2}{*}{ Classe } & \multicolumn{2}{c}{ KM } & & \multicolumn{2}{c}{ FCM } \\
\cline { 2 - 3 } \cline { 5 - 6 } & Médias & № pontos & & Médias & № pontos \\
1 & 58,6 & 25 & & 58,6 & 25 \\
2 & 61,8 & 48 & & 61,8 & 47 \\
3 & 64,1 & 27 & & 64,1 & 28 \\
\hline
\end{tabular}

Pode-se observar, na Tabela 3. que os métodos KM e FCM forneceram os mesmos valores de centroides para cada uma das classes; quanto ao número de pontos da grade padrão, houve diferença de apenas um ponto para as classes dois e três.

Na Figura 2 são apresentadas as zonas de manejo geradas a partir das concentrações foliares de $\mathrm{N}$, geradas pelos métodos KM e FCM.

A.

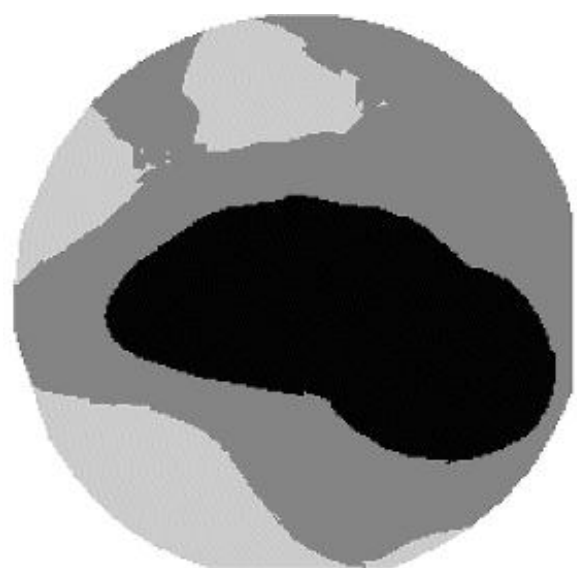

B.

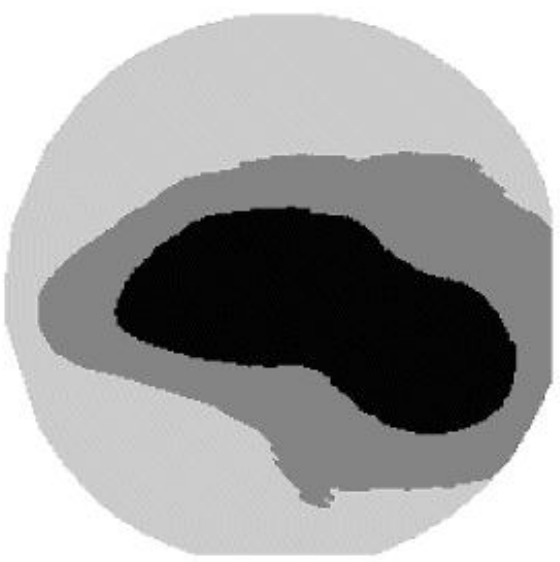

Classes

Figura 2. Zonas de manejo geradas a partir das concentrações foliares de $N$, pelos métodos $K M(A)$ e FCM (B) 
Na Tabela 4 se acham os valores médios de $\mathrm{N}$ que cada classe representa nas zonas de manejo geradas para os métodos KM e FCM e quantos pontos da grade padrão de 100 pontos pertencem a cada grupo.

Tabela 4. Valores médios de $\mathrm{N}$ e número de pontos da grade padrão de cada classe das zonas de manejo obtidas pelos métodos KM e FCM

\begin{tabular}{cccccc}
\hline \multirow{2}{*}{ Classe } & \multicolumn{2}{c}{ KM } & & \multicolumn{2}{c}{ FCM } \\
\cline { 2 - 3 } \cline { 5 - 6 } & Média & № pontos & & Média & № pontos \\
1 & 3,17 & 57 & & 3,19 & 44 \\
2 & 3,00 & 38 & & 3,05 & 43 \\
3 & 2,90 & 5 & & 2,93 & 13 \\
\hline
\end{tabular}

Pode-se observar, na Tabela 4, que os métodos KM e FCM tenderam a apresentar médias aproximadas, relativamente maiores para o método FCM; quanto ao número de pontos da grade padrão, ocorreu diferença de 13 pontos para a classe um, de 5 pontos para a classe dois e 8 pontos para a classe três, entre os métodos; após a comparação com as zonas de manejo geradas a partir dos valores de SPAD é possível saber qual dos dois métodos apresentou maior semelhança com o mapa de SPAD.

Na Figura 3 são apresentadas as zonas de manejo geradas a partir das concentrações foliares de N, P e K, pelos métodos KM e FCM.

A.

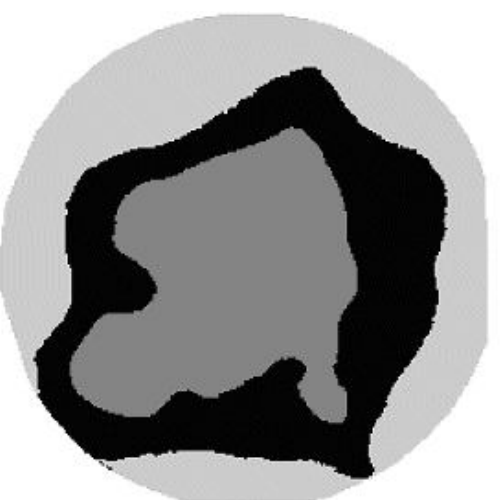

B.

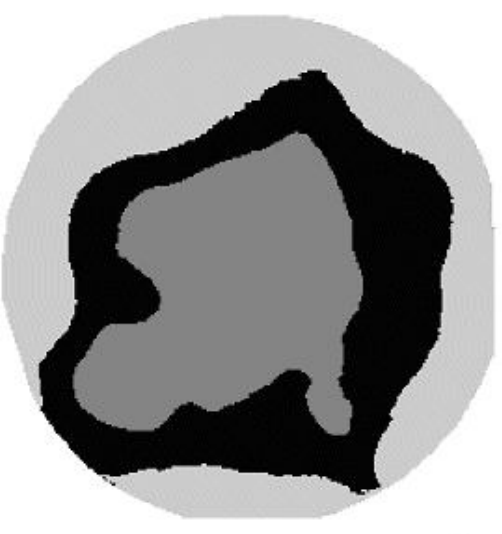

Classes

Figura 3. Zonas de manejo geradasa partir das concentrações foliares de N, P e K, pelos métodos KM (A) e FCM (B)
Apresentam-se, na Tabela 5, os valores de N, P e K que cada classe representa nas zonas de manejo geradas para os métodos KM e FCM e quantos pontos da grade padrão de 100 pontos são pertencentes a cada grupo.

Tabela 5. Valores médios de N, P e K e número de pontos da grade padrão de cada classe das zonas de manejo obtidas pelos métodosKM e FCM

\begin{tabular}{|c|c|c|c|c|c|c|c|c|}
\hline \multirow{3}{*}{ Classe } & \multicolumn{4}{|c|}{ KM } & \multicolumn{4}{|c|}{ FCM } \\
\hline & \multicolumn{3}{|c|}{ Média } & \multirow{2}{*}{$\begin{array}{c}\text { № } \\
\text { pontos }\end{array}$} & \multicolumn{3}{|c|}{ Média } & \multirow{2}{*}{$\begin{array}{c}\text { № } \\
\text { pontos }\end{array}$} \\
\hline & $\mathrm{N}$ & $\mathbf{P}$ & $K$ & & $\mathrm{~N}$ & $\mathbf{P}$ & K & \\
\hline 1 & 3,05 & 0,16 & 1,97 & 40 & 3,05 & 0,16 & 1,97 & 41 \\
\hline 2 & 3,04 & 0,15 & 2,23 & 54 & 3,04 & 0,15 & 2,23 & 53 \\
\hline 3 & 3,00 & 0,16 & 1,72 & 6 & 3,00 & 0,16 & 1,72 & 6 \\
\hline
\end{tabular}

Pode-se observar, na Tabela 5, que os métodos KM e FCM forneceram os mesmos valores de centroides para cada uma das classes; o único atributo que influenciou na delimitação das classes obtendo-se diferentes centróides, foi o K; quanto ao número de pontos da grade padrão houve diferença apenas de um ponto das classes um e dois, entre os métodos.

Na Figura 4 estão as zonas de manejo geradas a partir das concentrações foliares de $\mathrm{N}$ e Ca, pelos métodos KM e FCM.

A.

B.
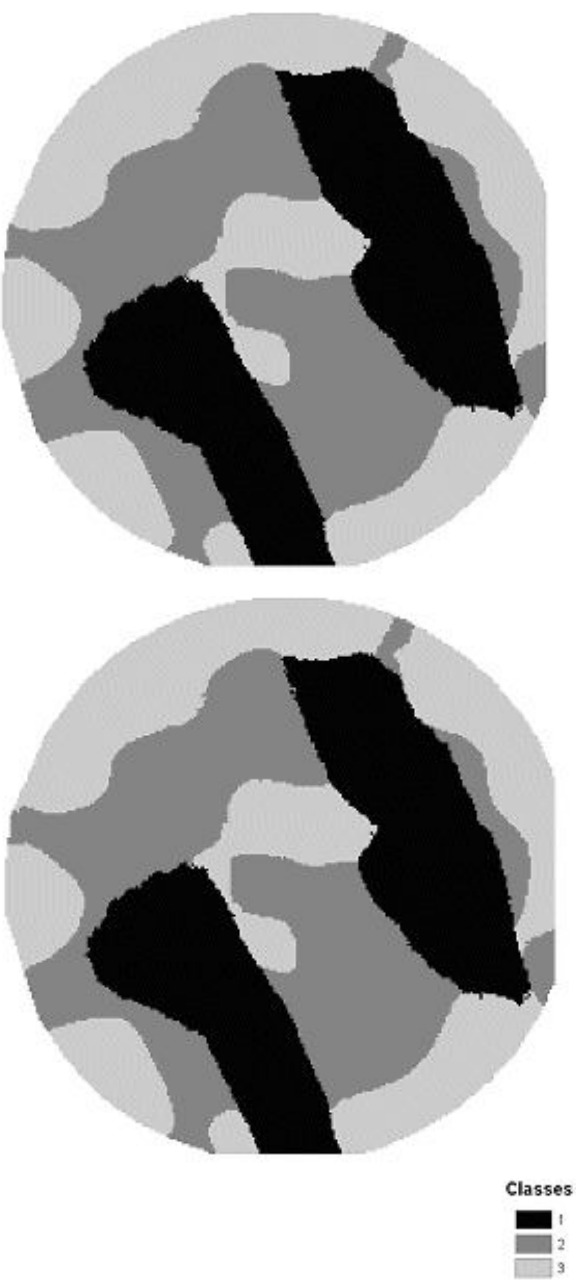

Figura 4. Zonas de manejo geradasa partir das concentrações foliares de $\mathrm{N}$ e $\mathrm{Ca}$, pelos métodos KM (A) e FCM (B) 
Tem-se, na Tabela 6, os valores de $\mathrm{N}$ e Ca que cada classe representa nas zonas de manejo geradas pelos métodos KM e FCM e quantos pontos da grade padrão de 100 pontos pertencem a cada grupo.

Tabela 6. Valores médios de $\mathrm{N}$ e Ca e número de pontos da grade padrão de cada classe das zonas de manejo, obtidas pelos métodos KM e FCM

\begin{tabular}{|c|c|c|c|c|c|c|}
\hline \multirow{3}{*}{ Classe } & \multicolumn{3}{|c|}{ KM } & \multicolumn{3}{|c|}{ FCM } \\
\hline & \multicolumn{2}{|c|}{ Média } & \multirow{2}{*}{$\begin{array}{c}\text { № } \\
\text { pontos }\end{array}$} & \multicolumn{2}{|c|}{ Média } & \multirow{2}{*}{$\begin{array}{c}\text { № } \\
\text { pontos }\end{array}$} \\
\hline & $\mathbf{N}$ & $\mathrm{Ca}$ & & $\mathbf{N}$ & $\mathrm{Ca}$ & \\
\hline 1 & 3,17 & 0,80 & 42 & 3,17 & 0,80 & 42 \\
\hline 2 & 2,97 & 0,82 & 39 & 2,97 & 0,83 & 39 \\
\hline 3 & 2,98 & 1,00 & 19 & 2,98 & 1,00 & 19 \\
\hline
\end{tabular}

Observa-se, na Tabela 6, que os métodos KM e FCM tenderam a apresentar os mesmos valores de centroides para cada uma das classes; o $\mathrm{N}$ influenciou na delimitação das classes um e dois, variando o valor dos centroides para essas classes; já para a classe três o Ca teve influência haja vista que $\mathrm{N}$ repetiu o valor de centróide da classe três; referente ao número de pontos da grade padrão, não houve diferença entre os métodos.

Na Figura 5 estão as zonas de manejo geradas a partir das concentrações foliares de $\mathrm{N}, \mathrm{Zn}$ e $\mathrm{B}$, pelos métodos KM e FCM.

A.

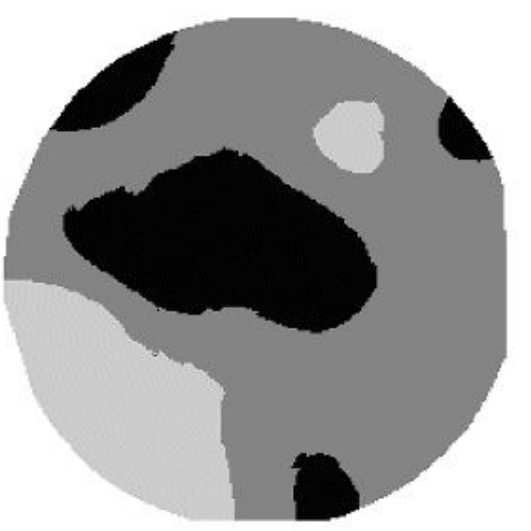

B.

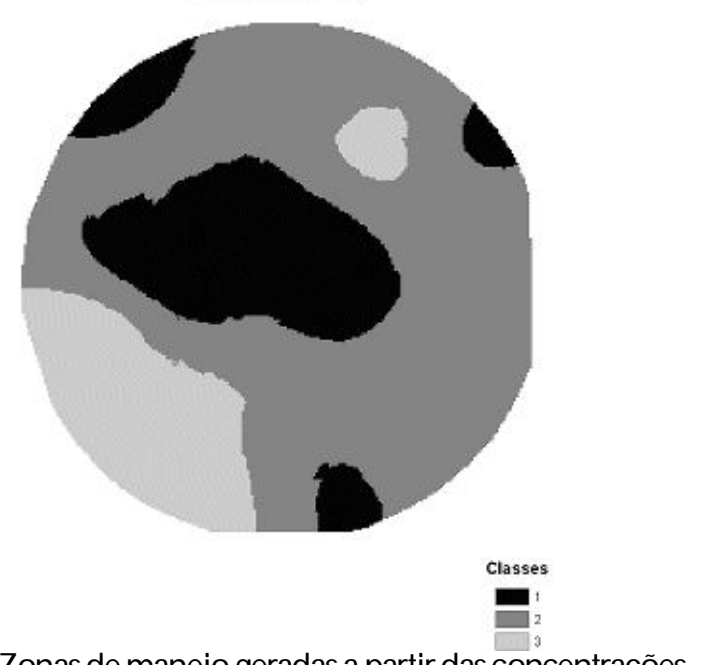

Figura 5. Zonas de manejo geradasa partir das concentrações foliares de N, Zn e B, pelos métodos KM (A) e FCM (B)
Na Tabela 7 se acham os valores de N, Zn e B, que cada classe representa nas zonas de manejo geradas para os métodos KM e FCM, e quantos pontos da grade padrão de 100 pontos pertencem a cada grupo.

Tabela 7. Valores médios de $\mathrm{N}, \mathrm{Zn}$ e $\mathrm{B}$ e número de pontos da grade padrão de cada classe das zonas de manejo, obtidas pelos métodos KM e FCM

\begin{tabular}{|c|c|c|c|c|c|c|c|c|}
\hline \multirow{3}{*}{ Classe } & \multicolumn{4}{|c|}{ KM } & \multicolumn{4}{|c|}{ FCM } \\
\hline & \multicolumn{3}{|c|}{ Média } & \multirow{2}{*}{$\begin{array}{c}\text { № } \\
\text { pontos }\end{array}$} & \multicolumn{3}{|c|}{ Média } & \multirow{2}{*}{$\begin{array}{c}\text { № } \\
\text { pontos }\end{array}$} \\
\hline & $\mathbf{N}$ & $\mathrm{Zn}$ & B & & $\mathrm{N}$ & $\mathrm{Zn}$ & B & \\
\hline 1 & 3,03 & 8,96 & 34,42 & 33 & 3,03 & 8,96 & 34,43 & 33 \\
\hline 2 & 3,05 & 8,43 & 29,47 & 55 & 3,05 & 8,43 & 29,47 & 55 \\
\hline 3 & 2,95 & 8,76 & 23,26 & 12 & 2,95 & 8,76 & 23,26 & 12 \\
\hline
\end{tabular}

Tal como nas situações anteriores, com exceção das zonas de manejo do $\mathrm{N}$ pode-se observar, na Tabela 7 , que os métodos KM e FCM tenderam a apresentar os mesmos valores de centroides para cada uma das classes; o B foi o atributo que tendeu a apresentar classes com centroides diferentes, tornando-o fator principal para a delimitação das mesmas nesse conjunto de dados; em referência ao número de pontos da grade padrão, não houve diferença nos pontos.

Na Figura 6 se apresentam as zonas de manejo geradas a partir das concentrações foliares de N, P, K, Ca e S, pelos métodos KM e FCM.

A.

B.
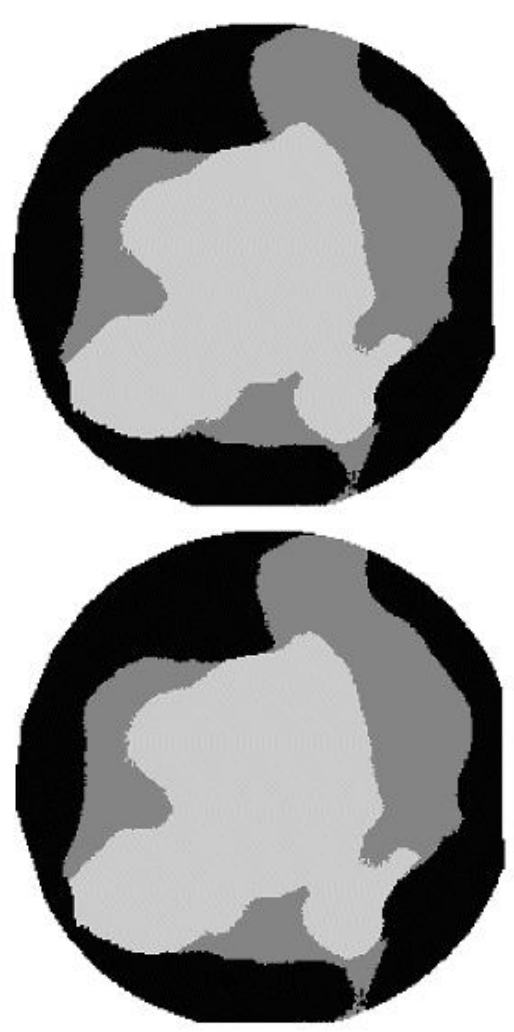

Classes

Figura 6. Zonas de manejo geradas a partir das concentrações foliares de N, P, K, Ca e S, pelos métodos KM (A) e FCM (B) 
Tabela 8. Valores médios de N, P, K, Ca e S e número de pontos da grade padrão de cada classe das zonas de manejo, obtidas pelos métodos KM e FCM

\begin{tabular}{|c|c|c|c|c|c|c|c|c|c|c|c|c|}
\hline \multirow{3}{*}{ Classe } & \multicolumn{6}{|c|}{ KM } & \multicolumn{6}{|c|}{ FCM } \\
\hline & \multicolumn{5}{|c|}{ Média } & \multirow{2}{*}{$\begin{array}{c}\text { № } \\
\text { pontos }\end{array}$} & \multicolumn{5}{|c|}{ Média } & \multirow{2}{*}{$\begin{array}{c}\text { № } \\
\text { pontos }\end{array}$} \\
\hline & $\mathrm{N}$ & $\mathbf{P}$ & $K$ & $\mathrm{Ca}$ & $S$ & & $\mathbf{N}$ & $\mathbf{P}$ & $K$ & $\mathrm{Ca}$ & $S$ & \\
\hline 1 & 2,98 & 0,16 & 1,74 & 0,95 & 0,15 & 7 & 2,98 & 0,16 & 1,74 & 0,95 & 0,15 & 6 \\
\hline 2 & 3,11 & 0,15 & 1,93 & 0,80 & 0,16 & 30 & 3,11 & 0,15 & 1,93 & 0,80 & 0,16 & 30 \\
\hline 3 & 3,03 & 0,15 & 2,20 & 0,88 & 0,17 & 63 & 3,03 & 0,15 & 2,20 & 0,88 & 0,17 & 64 \\
\hline
\end{tabular}

Na Tabela 8 estão os valores de N, P, K, Ca e S que cada classe representa nas zonas de manejo geradas para os métodos KM e FCM e quantos pontos da grade padrão de 100 pontos fazem parte de cada grupo.

Observa-se, na Tabela 8, que os métodos KM e FCM forneceram os mesmos valores de centroides para cada uma das classes; o $\mathrm{K}$ foi o atributo que tendeu a apresentar maior diferença para os centroides, nas três classes; quanto ao número de pontos da grade padrão, houve diferença apenas de um ponto das classes um e três, entre os métodos.

$\mathrm{Na}$ Figura 7 se apresentam as zonas de manejo geradas a partir das concentrações foliares de N, Ca e S, pelos métodos KM e FCM.

A.

B.

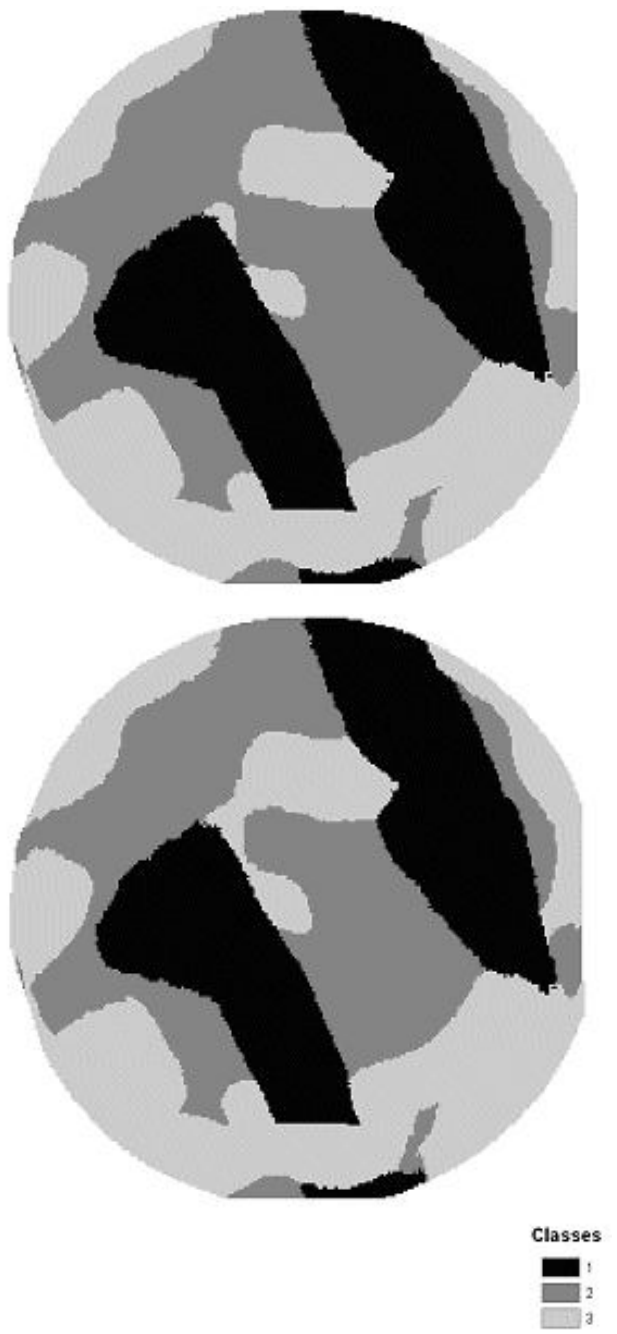

Figura 7. Zonas de manejo geradasa partir das concentrações foliares de N, Ca e S, pelos métodos KM (A) e FCM (B)
Na Tabela 9 são apresentados os valores de N, Ca e S que cada classe representa nas zonas de manejo geradas para os métodos KM e FCM, e quantos pontos da grade padrão de 100 pontos são pertencentes a cada grupo.

Tabela 9. Valores médios de N, Ca e S e número de pontos da grade padrão de cada classe das zonas de manejo, obtidos pelos métodos KM e FCM

\begin{tabular}{|c|c|c|c|c|c|c|c|c|}
\hline \multirow{3}{*}{ Classe } & \multicolumn{4}{|c|}{ KM } & \multicolumn{4}{|c|}{ FCM } \\
\hline & \multicolumn{3}{|c|}{ Média } & \multirow{2}{*}{$\begin{array}{c}\text { № } \\
\text { pontos }\end{array}$} & \multicolumn{3}{|c|}{ Média } & \multirow{2}{*}{$\begin{array}{c}\text { № } \\
\text { pontos }\end{array}$} \\
\hline & $\mathrm{N}$ & $\mathrm{Ca}$ & $S$ & & $\mathbf{N}$ & $\mathrm{Ca}$ & $S$ & \\
\hline 1 & 3,16 & 0,80 & 0,16 & 41 & 3,17 & 0,80 & 0,16 & 41 \\
\hline 2 & 2,97 & 0,83 & 0,16 & 38 & 2,97 & 0,83 & 0,16 & 37 \\
\hline 3 & 2,98 & 1,01 & 0,15 & 21 & 2,98 & 1,01 & 0,15 & 22 \\
\hline
\end{tabular}

Novamente se observa, na Tabela 9, que os métodos KM e FCM tenderam a apresentar os mesmos valores de centroides para cada uma das classes; o $\mathrm{N}$ influenciou na delimitação das classes um e dois; já para a classe três o Ca exerceu maior influência; o $S$ permaneceu praticamente o mesmo para as três classes indicando que a delimitação das classes se deu com base nos valores de $\mathrm{N}$ e Ca; quanto aos números de pontos da grade padrão, houve diferença de apenas um ponto das classes dois e três, entre os métodos.

Analisando os resultados apresentados nas Figuras 4 e 7 , pode-se observar que as zonas de manejo pouco diferenciaram; o centroide para o elemento S não alterou para as três classes; sendo assim, o mesmo não influenciou na sua delimitação.

Na Tabela 10, a seguir, são apresentadas as porcentagens de pontos que foram dispostos em mesma classe, entre as zonas de manejo geradas a partir das concentrações foliares e as zonas de manejo geradas com os valores de SPAD, individualmente, para cada método utilizado.

Tabela 10. Porcentagem de pontos de mesma classe entre as zonas de manejo do SPAD e as zonas de manejo das concentrações foliares

\begin{tabular}{lcccccc}
\hline \multirow{2}{*}{ Método } & \multicolumn{6}{c}{ Zonas de manejo } \\
\cline { 2 - 8 } & $\mathbf{N}, \mathbf{P}, \mathbf{K}$ & $\mathbf{N}, \mathbf{C a}$ & $\mathbf{N}$ & $\mathbf{N}, \mathbf{Z n}, \mathbf{B}$ & $\mathbf{N}, \mathbf{P}, \mathbf{K}, \mathbf{C a}, \mathbf{S}$ & $\mathbf{N}, \mathbf{C a}, \mathbf{S}$ \\
KM & 33 & 31 & 38 & 38 & 18 & 39 \\
FCM & 33 & 29 & 34 & 37 & 18 & 38 \\
\hline
\end{tabular}

Como indicado na Tabela 10, todos os conjuntos de dados utilizados na geração das zonas de manejo forneceram porcentagens abaixo de $39 \%$ de pontos coincidentes da mesma classe, em comparação com as zonas de manejo de SPAD, para os dois métodos; para os mapas de $\mathrm{N}$, os únicos que apresentaram diferença entre os métodos, o método KM foi o 
que forneceu maior porcentagem de pontos coincidentes de mesma classe com o mapa de SPAD.

Diferentes fontes de dados podem ser usadas para a delimitação de zonas de manejo. Exemplos na literatura abrangem desde a utilização do conhecimento do agricultor (Fleming \& Westfall, 2000), dados de produtividade (Diker et al., 2004; Brock et al., 2005; Flowers et al., 2005; Jaynes et al., 2005), amostragem de solo (Franzen et al., 2002; Vrindts et al., 2005), imagens (Stewart \& Mcbratney, 2001; Taylor et al., 2002), sensores de indução eletromagnética e condutividade elétrica aparente (Hedley et al., 2004; Li et. al., 2008), espectrometria gama (Wong et al., 2008), até a combinação de alguns desses dados (Whelan et al., 2002; Godwin et al., 2003; Taylor et al., 2003; Fleming et al., 2004; Koch et al., 2004; Mallarino \& Wittry, 2004; Schepers et al., 2005; Simbahan \& Dobermann, 2006; Kyaw et al., 2008). O SPAD pode mostrar-se adequado para gerar zonas de manejo do estado nutricional da lavoura, ou seja, pode-se delimitar zonas melhor nutridas que outras mas não se pode dizer qual é o nutriente envolvido.

Segundo Doerge (1999), há diversas técnicas para a delineação de zonas de manejo propostas na literatura; o que todas as técnicas descrevem em comum é que elas são de fácil aplicação, indicando não utilizar exclusivamente amostragem de solo como fator de determinação das zonas de manejo possibilitando, desta forma, o uso de diversos fatores agronômicos em conjunto; portanto, têm potencial de serem economicamente mais viáveis do que a grade de amostragem necessária para aplicações a taxas variáveis. Após todas as comparações das zonas de manejo geradas com cada grupo pelos dois métodos, é possível afirmar que ambos os métodos tenderam a apresentar agrupamentos similares, permitindo a escolha de qualquer um dos dois métodos.

É válido salientar que a área em estudo é relativamente pequena (2,10 ha), em comparação com outros trabalhos encontrados na literatura de geração de zonas de manejo; também não apresenta muita variabilidade pois é devidamente adubada e não possui stress hídrico, em virtude do sistema de irrigação.

Segundo Ortega \& Santibáñez (2007), para a delimitação de zonas de manejo é mais importante identificar as variáveis do solo responsáveis pela variação da produtividade do que selecionar o método mais robusto para fazer tais delimitações. É difícil determinar que informações devem ser usadas na delimitação de zonas de manejo de uma área; sugere-se, então, a utilização de atributos isolados ou combinados que reflitam o comportamento do objeto em que se deseja caracterizar a variabilidade. Quanto maior a correlação entre os atributos utilizados na geração das zonas de manejo e o objeto do qual se deseja caracterizar, maior também será a confiabilidade dos mapas gerados com os atributos.

\section{Conclusões}

A utilização dos métodos K-Means e Fuzzy C-Means permitiram a geração de zonas de manejo com base nos valores de SPAD e análise foliar. Tendo em vista os resultados obtidos, pode-se concluir que:
1. Os métodos de agrupamento de dados K-Means e Fuzzy C-Means não apresentaram diferenças na geração das zonas de manejo.

2. Houve similaridades variando de 18 a $39 \%$ entre as zonas de manejo de SPAD e os diferentes grupos de concentrações de nutrientes foliares.

\section{LITERATURA CITADA}

Ateca, M. R.; Sereno, R.; Apezteguía, H. Zonificacion de una superficie cultivada com soja segun aspectos fenométricos y consumo de agua del suelo. Revista Brasileira de Agrometeorologia, v.9, p.111-116, 2001.

Brock, A.; Brouder, S. M.; Blumhoff, G.; Hofmann, B. S. Defining yield-based management zones for corn-soybean rotations. Agronomy Journal, v.97, p.1115-1128, 2005.

Diker, K.; Heerman, D. F.; Brodahl, M. K. Frequency analysis of yield for delineating yield response zones. Precision Agriculture, v.5 p.435-444, 2004.

Doerge, T. Management zone concepts. SSMG-2. In: Site specific management guidelines. Norcross: Potash and Phosphate Institute, 1999. 4p.

Fleming, K. L.; Heerman, D. F.; Westfall, D. G. Evaluating soil color with farmer input and apparent soil electrical conductivity for management zone delineation. Agronomy Journal, v.96, p.1581-1587, 2004.

Fleming, K. L.; Westfall, D. G. Evaluating farmer defined management zone maps for variable rate fertilizer application. Precision Agriculture, v.2, p.201-215, 2000.

Flowers, M.; Weisz, R.; White, J. G. Yield-based management zones and grid sampling strategies: Describing soil tests and nutrient availability. Agronomy Journal, v.97, p.968982, 2005.

Franzen, D. W.; Hopkins, D. H.; Sweeney, M. D.; Ulmer, M. K.; Halvorson, A. D. Evaluation of soil survey scale for zone development of site-specific nitrogen management. Agronomy Journal, v.94, p.381-389, 2002.

Godwin, R. J.; Wood, G. A.; Taylor, J. C.; Knight, S. M.; Welsh, J. P. Precision farming of cereal crops: a review of a six year experiment to develop management guidelines. Biosystems Engineering, v.84, p.375-391, 2003.

Hedley, C. B.; Yule, I. J.; Eastwood, C. R.; Shepherd, T. G.; Arnold, G. Rapid identification of soil textural and management zones using electromagnetic induction sensing of soil. Australian Journal of Soil Research, v.42, p.389-400, 2004.

Isaaks, E. H.; Srivastava, R. M. An introduction to applied geoestatistics. New York: Oxford Univertsity Press. 1989. $560 \mathrm{p}$.

Jaynes, D. B.; Colvin, T. S.; Kaspar, T. C. Identifying potential soybean management zones from multi-year yield data. Computers and Electronics in Agriculture, v.46, p.309-327, 2005.

Koch, B.; Khosla, R.; Frasier, W. M.; Westfall, D. G.; Inman, D. Economic feasibility of variable-rate nitrogen application utilizing site-specific management zones. Agronomy Journal, v.96, p.1572-1580, 2004. 
Kyaw, T.; Ferguson, R. B.; Adamchuk, V. I.; Marx, D. B.; Tarkalson, D. D.; McCallister, D. L. Delineating site-specific management zones for $\mathrm{pH}$-induced iron chlorosis. Precision Agriculture, v.9, p.71-84, 2008.

Lange, A.; Martines, A. M.; Silva, M. A. C.; Sorreano, M. C. M.; Cabral, C. P.; Malavolta, E. Efeito de deficiência de micronutrientes no estado nutricional da mamoneira cultivar Iris. Pesquisa Agropecuária Brasileira, v.40, p.61-67, 2005.

Li, Y.; Shi, Z.; Wu, C.; Li, H.; Li, F. Determination of potential management zones from soil electrical conductivity, yield and crop data. Journal of Zhejiang University Science B, v.9, p.68-76, 2008.

Mallarino, A. P.; Wittry, D. J. Efficacy of grid and zone soil sampling approaches for site-specific assessment of phosphorus, potassium, $\mathrm{pH}$, and organic matter. Precision Agriculture, v.5, p.131-144, 2004.

Martinez, H. E. P.; Menezes, J. F. S.; Souza, R. B.; Venegas, V. H.; Guimarães, P. T. G. Faixas críticas de concentrações de nutrientes e avaliação do estado nutricional de cafeeiros em quatro regiões e Minas Gerais. Pesquisa Agropecuária Brasileira, v.38, p.703-713, 2003.

Minasny, B.; Mcbratney, A. B. FuzME version 3.0, Australian Centre for Precision Agriculture, Sydney: The University of Sydney, 2002.

Ortega, R. A.; Santibáñez, O. A. Determination of management zones in corn (Zea mays L.) based on soil fertility. Computers and Electronics in Agriculture, v.58, p.49-59, 2007.

Queiroz, D. M.; Pinto, F. A. C.; Zandonadi, R. S.; Emerich, I. N.; Sena Júnior, D. G. Uso de técnicas de agricultura de precisão para a cafeicultura de montanha. In: Zambolim, L. Efeitos da irrigação sobre a qualidade e produtividade do café. Viçosa: Editora UFV, 2004. Cap.3, p.77-108.

Santos, A. O.; Pallone Filho, W. J.; Ungaro, M. R. G.; Lino, A. C. L.; Radin, B. Prospecção de zonas potenciais para manejo diferenciado em agricultura de precisão utilizando-se padrões de solo-planta-clima. Revista Brasileira de Engenharia Agrícola e Ambiental, v.7, p.463-468, 2003.

Schepers, A. R.; Shanahan, J. F.; Liebig, M. A.; Schepers, J. S.; Johnson, S. H.; Luchiaro, A. Appropriateness of management zones for characterizing spatial variability of soil properties and irrigated corn yields across years. Agronomy Journal, v.96, p.195-203, 2005.

Simbahan, G. C.; Dobermann, A. Sampling optimization based on secondary information and its utilization in soil carbon mapping. Geoderma, v.133, p.345-362, 2006.
Stewart, C. M.; Mcbratney, A. B. Using bare soil imagery to determine management zones for the variable-rate application of inputs for cotton. In: Grenier, G.; Blackmore, S. Proceedings of the 3rd European Conference on Precision Agriculture. Montpellier: Agro-Montpellier ENSAM, 2001. p.319-324.

Taylor, J. A.; Mcbratney, A. B.; Ciavarella, R. Predicting and mapping winegrape quality from multiple must properties. In: Robert, P. C.; Rust, R. H.; Larson, W. E. Proceedings of the 6th International Conference on Precision Agriculture. Minneapolis: ASA, CSSA, SSSA, Madison, WI. 2002. p.614623.

Taylor, J. A.; Mcbratney, A. B.; Whelan, B. M. Establishing management classes for broadacre agricultural production. Agronomy Journal, v.99, p.1366-1376, 2007.

Taylor, J. C.; Wood, G. A.; Earl, R.; Godwin, R. J. Soil factors and their Inûuence on Within-ûeld Crop Variability, Part II: Spatial Analysis and Determination of Management Zones. Biosystems Engineering, v.4, p.441-453, 2003.

Vrindts, E.; Mouazen, A. M.; Reyniers, M.; Maertens, K.; Maleki, M. R.; Ramon, H.; Baerdemaeker, J. Management Zones based on correlation between soil compaction, yield and crop data. Biosystems Engineering, v.92, p.419-428, 2005.

Windham, M. P. Cluster validity c-means for fuzzy clustering algorithm. IEEE Transactions. Pattern Analyses Machine Intelligence, v.4, p.354-363, 1982.

Whelan, B. M.; Cupitt, J.; Mcbratney, A. B. Practical definition and interpretation of potential management zones in Australian dryland cropping. In: Robert, P. C.; Rust, R. H.; Larson, W. E. Proceedings of the 6th International Conference on Precision Agriculture. Minneapolis: ASA, CSSA, SSSA, Madison, WI. 2002. p.315-329.

Wong, M. T. F.; Asseng, S.; Robertson, M. J.; Oliver, Y. Mapping subsoil acidity and shallow soil across a field with information from yield maps, geophysical sensing and the grower. Precision Agriculture, v.9, p.3-15, 2008.

Yan, L.; Zhou, S.; Feng, L.; Hong-Yi, L. Delineation of sitespecific management zones using fuzzy clustering analysis in a coastal saline land. Computers and Electronics in Agriculture, p.174-186, 2007.

Yonamine, F. S.; Specia, L.; Carvalho, V. O.; Nicoletti, M. C. Aprendizado não supervisionado em domínios fuzzy Algoritmo fuzzy c-means. São Carlos: UFSCAR, 2002. 18p.

Zimback, C. R. L. Análise espacial de atributos químicos de solos para fins de mapeamento da fertilidade. Botucatu: FCA/UNESP, 2001. 114p. Tese Livre-Docência 